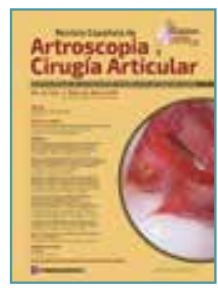

\title{
Originales
}

\section{¿Existe relación entre el ángulo crítico del hombro y los resultados funcionales tras la reparación del manguito rotador? Resultados preliminares}

\author{
C. I. Palomo', C. Oliveira², D. Gómez³ , M. A. Hernán ${ }^{4}$ \\ ${ }^{1}$ Servicio de Traumatología y Cirugía Ortopédica. Hospital Nuestra Señora del Rosario. Madrid \\ 2 Servicio de Traumatología y Cirugía Ortopédica. Unidade Local de Saúde do Alto Minho. \\ Viana do Castelo. Portugal \\ ${ }^{3}$ Servicio de Traumatología y Cirugía Ortopédica. Hospital General de Villalba. Madrid \\ ${ }^{4}$ Unidad de Artroscopia. Hospital Universitario Santa Cristina. Madrid
}

Correspondencia:

Dr. Carlos Iván Palomo Pizarro

Correo electrónico: carlos_cip23@hotmail.com
Recibido el 30 de mayo de 2017

Aceptado el 5 de febrero de 2017

Disponible en Internet: marzo de 2018

\section{RESUMEN}

Objetivo: el ángulo crítico del hombro es una medida radiológica que se ha relacionado con la patología degenerativa del manguito rotador. El objetivo fue investigar la relación existente entre este ángulo y los resultados funcionales tras la reparación artroscópica de las roturas del manguito rotador.

Métodos: estudio retrospectivo sobre 100 pacientes intervenidos por roturas degenerativas completas del manguito rotador. Las variables evaluadas fueron: ángulo crítico del hombro preoperatorio, edad, lateralidad, tipo de rotura, tipo de reparación, complicaciones y el test de Constant preoperatorio, al mes, 3 y 6 meses postoperatorio.

Resultados: la media del ángulo crítico fue de 37,19 $\pm 3,12^{\circ}$ $\left(32-46^{\circ}\right)$ (prueba de Kolmogorov-Smirnov). El test de Constant preoperatorio obtuvo una media de 52,18 \pm 11 , al mes de 54,20 $\pm 9,50$ (25-80), a los 3 meses de 65,86 $\pm 8,96$ (30$85)$ y a los 6 meses de 79,58 $\pm 8,86$ (40-95). Se encontró una relación estadísticamente significativa $(p<0,05)$ entre el tamaño de la rotura y el Constant preoperatorio $(p=0)$ y al mes $(p=0,013)$ (prueba Ty de Mann-Whitney). Se observó

\begin{abstract}
Is there an association between critical shoulder angle and functional outcomes after rotator cuff repair? Preliminary results
\end{abstract}

Objective: critical shoulder angle (CSA) is a radiographic parameter which has been associated with degenerative tears of the rotator cuff. The purpose was to evaluate the relationship between the CSA and the functional outcomes after arthroscopic rotator cuff repair.

Materials and methods: this was a retrospective review of 100 patients with full-thickness tears who had undergone arthroscopic rotator cuff repair. Preoperative $X$-rays were used to measure the CSA. Tear size was determined intraoperatively. Constant score was assessed preoperatively, at 1 month, 3 months and 6 months. A single-row repair was done in all patients.

Results: average CSA was $37,19 \pm 3,12^{\circ}\left(32-46^{\circ}\right)$ (Kolmogorov-Smirnov test). Constant score preoperatively was $52,18 \pm 11$, at 1 month 54,20 $\pm 9,50(25-80), 3$ months $65,86 \pm 8,96$ (30-85) and 6 months 79,58 $\pm 8,86$ (40-95). A statistically relevant rela-

https://doi.org/10.24129/j.reaca.25161.fs1705019

FS $@ 2018$ Fundación Española de Artroscopia. Publicado por Imaidea Interactiva en FONDOSCIENCE ${ }^{\circledR}$ (www.fondoscience.com). Este es un artículo Open Access bajo la licencia CC BY-NC-ND (www.creativecommons.org/licenses/by-nc-nd/4.0/). 
una asociación no significativa entre puntuaciones mayores en la escala de constant y valores de ángulo crítico menores al primer mes $(p=0,699)$ y al tercero $(p=0,764)$ (test de Pearson). Existió un porcentaje de incidencias postoperatorias del $14,7 \%$.

Conclusiones: un valor elevado del ángulo crítico se relaciona con mayor riesgo de lesión del manguito al compararlo con otras publicaciones. Existe una asociación significativa entre el tamaño de la rotura y el test de Constant preoperatorio y al primer mes. No podemos confirmar una asociación significativa entre valores inferiores de ángulo crítico y valores superiores en la escala de Constant.

Nivel de evidencia: nivel III. Estudio de cohortes retrospectivo. Relevancia clínica: se desconoce cómo influye un ángulo crítico elevado tras la reparación del manguito y si la propia cirugía podría alterar la evolución natural de la recuperación.

Palabras clave: Ángulo crítico del hombro. Reparación manguito rotador. Resultados funcionales.

\section{Introducción}

Las lesiones degenerativas del manguito rotador se encuentran entre las patologías más frecuentes del hombro ${ }^{(1)} \mathrm{y}$, aunque existe controversia en cuanto a su patogenia, las variaciones en la morfología escapular han sido asociadas con su desarrollo(2). Diversas teorías recientes sugieren que existe una predisposición morfológica que alteraría la biomecánica del hombro y favorecería el desarrollo de estas roturas.

Varios estudios han demostrado que una mayor oblicuidad de la cavidad glenoidea se asocia con un incremento del componente de cizallamiento de las fuerzas generadas por el músculo deltoides ${ }^{(3-5)} y$, por tanto, con una mayor elevación de la cabeza humeral.

Además, una marcada extensión lateral del acromion aumenta el componente vertical de las fuerzas del deltoides ${ }^{(6-8)}$. Estas fuerzas verticales podrían incrementar la elevación de la cabeza humeral y con ello favorecer la lesión del manguito rotador por sobrecarga del mismo y por el desarrollo de impingement subacromial, sobre todo en aquellos ángulos mayores de $38^{\circ(9-11)}$.

Para evaluar estas fuerzas, Moor ${ }^{(6)}$ describió el ángulo crítico del hombro (Figura 1), que es una tion $(p<0.05)$ was found between the size of the tear and the preoperatory Constant $(p=0)$ and after one month $(p=0.013)$ ( $T$ and Mann-Whitney test). There was no significant difference between high Constant score values and low CSA values at 1 month $(p=0,699)$ and at 3 months $(p=0,764)$ (Pearson test). Postoperative incidences were $14,7 \%$.

Conclusion: higher CSA is correlated with high risk of rotator cuff tear when compared to other articles. There is a significant association between the size of the tear and preoperative Constant score and at 1 month. A relevant relation between low CSA values with high Constant values cannot be confirmed.

Level of evidence: level III. Retrospective cohort design.

Clinical relevance: it is unknown both the impact of high CSA after the repair of rotator cuff and if the surgery itself may alter the natural development of recovery.

Key words: Critical shoulder angle. Rotator cuff repair. Functional outcomes.

medida radiológica que está formada por una línea que conecta el margen inferior y el margen superior de la cavidad glenoidea y otra línea que une el margen inferior de la glenoides con el borde lateral del acromion, cuya ventaja es que combina la extensión lateral del acromion con la inclinación de la fosa glenoidea en una única medida cuantificable. De manera que las cifras $>35^{\circ}$ se relacionaron con el aumento del riesgo de lesión no traumática del manguito rotador y valores $<30^{\circ}$ con artrosis glenohumeral.

Se trata del predictor anatómico más potente de roturas degenerativas del manguito, de entre los parámetros radiológicos más estudiados (índice acromial, ángulo lateral del acromion, pendiente acromial, morfología acromial de Bigliani, etc.) $)^{(12,13)}$. Este ángulo presenta una concordancia interobservador excelente ${ }^{(13)}$ y es un predictor potente y sensible de roturas degenerativas tanto parciales como totales debido a que toma en cuenta ambos parámetros y a que son fácilmente medibles con exactitud en la radiografia ${ }^{(14,15)}$ y tomografía computarizada ${ }^{(16)}$, siendo más fiable que la medida obtenida mediante resonancia magnética $(R M)^{(17)}$. Sin embargo, es importante realizar una proyección anteroposterior (AP) verdadera de hombro, ya que se ha demostrado que desviaciones mayores de $5^{\circ}$ 


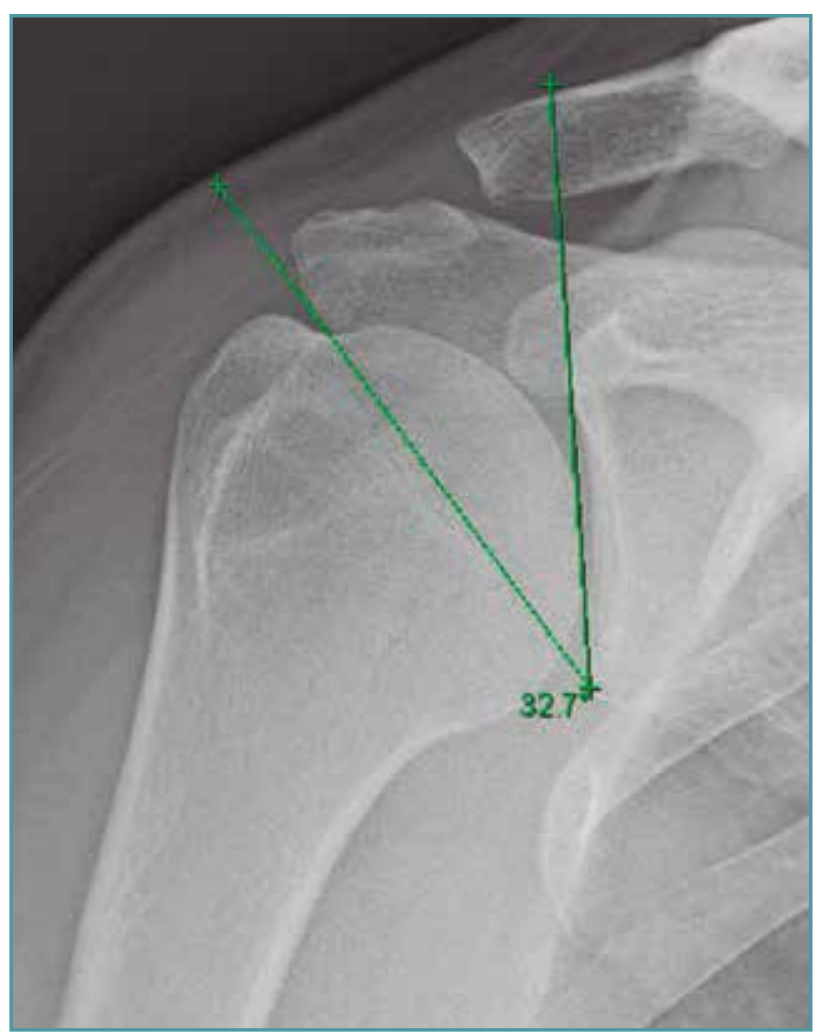

Figura 1. Radiografía con proyección anteroposterior verdadera de hombro derecho. Medición del ángulo crítico del hombro $\left(32,7^{\circ}\right)$.

en la anteversión pueden resultar en más de $2^{\circ}$ de diferencia en el valor del ángulo crítico ${ }^{(15)}$.

En el estudio radiográfico y sobre tomografía computarizada de Daggett ${ }^{(18)}$, también se concluyó que la inclinación glenoidea está íntimamente relacionada con el ángulo crítico del hombro y se encuentra significativamente incrementada en pacientes con roturas masivas del manguito rotador.

El objetivo de nuestro estudio fue investigar la relación existente entre el ángulo crítico del hombro y los resultados funcionales tras la reparación artroscópica de las roturas completas degenerativas del manguito rotador, con la hipótesis de que en aquellos pacientes con un ángulo crítico menor se obtendrían mejores valores funcionales postoperatorios, pudiendo ser útil como factor pronóstico.

En el momento actual, solo hemos encontrado en la bibliografía un artículo de García et al.(19) y otro de Kirsch et al. ${ }^{(20)}$ en los que se comparan ambos parámetros.

\section{Métodos}

Se realizó un estudio retrospectivo revisando a 100 pacientes intervenidos por roturas degenerativas completas del manguito rotador, mediante reparación artroscópica y acromioplastia durante los años 2013 y 2014, tras aceptar el consentimiento informado, con un periodo mínimo de seguimiento de 6 meses.

Los criterios de inclusión fueron los siguientes: rotura del manguito rotador confirmada por RM, proyección AP verdadera de hombro preoperatoria con solapamiento de borde anterior y posterior de la glenoides $<5 \mathrm{~mm}$ y ausencia de traumatismo ni cirugía previa del hombro. Todos los pacientes comenzaron rehabilitación progresiva controlada según la tolerancia desde el día después de la intervención.

Las variables estudiadas fueron: el ángulo crítico del hombro preoperatorio con radiografía simple, la edad, la lateralidad, el tipo de rotura, el tipo de reparación, el test de Constant preoperatorio, al mes, 3 y 6 meses postoperatorio. De igual modo, se valoraron las complicaciones.

Las cirugías fueron realizadas por un único cirujano experto de hombro y las medidas radiológicas y clínicas fueron recogidas por 3 médicos internos residentes de manera ciega.

Se realizó el análisis estadístico mediante el programa SPSS 15.0 (SPSS, Chicago, IL, USA) por un estadístico independiente, empleándose la prueba de Kolmogorov-Smirnov para evaluar la normalidad de las variables, el test de Pearson, la prueba T y la prueba de Mann-Withney, estableciéndose la significación estadística en $p<0,05$.

\section{Resultados}

Cumplieron los criterios de inclusión 83 pacientes, valorándose finalmente 88 hombros y apreciándose los resultados que se detallan a continuación.

Mediante la prueba de Kolmogorov-Smirnov se obtuvo una media del ángulo crítico de $37,1 \pm 3,1^{\circ}\left(32-46^{\circ}\right)$. El test de Constant preoperatorio obtuvo una media de $52,18 \pm 11$, al mes de $54,20 \pm 9,50$ (25-80), a los 3 meses de 65,86 $\pm 8,96$ $(30-85)$ y a los 6 meses de 79,58 $\pm 8,86(40-95)$ (Tabla 1).

Con la prueba de Mann-Whitney y la prueba T se encontró una relación estadísticamente signi- 


\begin{tabular}{|c|c|c|c|}
\hline \multicolumn{4}{|c|}{$\begin{array}{l}\text { Tabla 1. Prueba de Kolmogorov-Smirnov para } \\
\text { calcular las medias y desviaciones } \\
\text { estándar del ángulo crítico y de las } \\
\text { puntuaciones en el test de Constant }\end{array}$} \\
\hline & Media & Desviación típica & $\mathbf{N}$ \\
\hline Ángulo crítico & 37,1932 & 3,12152 & 88 \\
\hline Constant preop. & 52,1818 & 11,00814 & 100 \\
\hline Constant 1 mes & 54,2041 & 9,50398 & 99 \\
\hline Constant 3 meses & 65,8632 & 8,96262 & 96 \\
\hline Constant 6 meses & 79,5889 & 8,86553 & 91 \\
\hline
\end{tabular}

La media del ángulo crítico obtenido $\left(37^{\circ}\right)$ es un valor elevado que se relaciona con mayor riesgo de sufrir lesiones del manguito rotador

ficativa entre el tamaño de la rotura y el Constant preoperatorio $(p=0)$ y al mes $(p=0,013)$, y prácticamente también a los 3 meses $(p=0,056)$.

Con el test de Pearson se observó, en valores de ángulo crítico menores, una tendencia a puntuaciones mayores en la escala de Constant en el preoperatorio $(p=0,658)$, al primer mes $(p=0,699)$, al tercero $(p=0,764)$ y al sexto $(p=0,654)$, sin ser la diferencia estadisticamente significativa.

En cuanto a los resultados demográficos, la edad media fue de 55,1 años, encontrándose 30 hombres (36,1\%) y 53 mujeres (63,9\%), con afectación del lado derecho en 62 casos $(70,5 \%)$ y del lado izquierdo en 26 pacientes (29,5\%). En cuanto a la etiología, el 4,55\% fue deportiva, laboral en el $48,86 \%$ y sin claro origen en el $46,59 \%$. El tipo de rotura se dividió según el tamaño, siendo pequeñas aquellas menores de $3 \mathrm{~cm}$ en el $70 \%$ y grandes mayores de $3 \mathrm{~cm}$ en el $30 \%$. Existió un porcentaje de incidencias postoperatorias del 14,7\% (rerrotura en el $9,09 \%$ de los pacientes, rigidez en un $3,41 \%$, dolor en la porción larga del bíceps que requirió tenotomía en el 1,14\% e inestabilidad en el 1,14\% de los pacientes). La tasa de seguimiento final fue del $100 \%$.

\section{Discusión}

El manguito rotador del hombro tiene la función de centralizar la cabeza humeral sobre la glenoides, neutralizando las fuerzas ascendentes del músculo deltoides. Con el incremento de la edad, así como con otros factores intrínsecos y extrín- secos, se produce una degeneración progresiva del mismo que va a provocar su debilidad para contrarrestar al deltoides, resultando en un ascenso de la cabeza humeral con un pinzamiento subacromial secundario y con mayor desgaste tendinoso. Además, cuanto mayor sea el valor del ángulo crítico del hombro, mayor será la carga de trabajo del manguito rotador para contrarrestar al músculo deltoides, acelerándose el proceso degenerativo y favoreciéndose la rotura del mismo. De manera similar, un traumatismo o múltiples microtraumatismos exacerbarían el proceso ${ }^{(13)}$.

Por ello, los pacientes que han sido sometidos a una reparación artroscópica y que tienen un ángulo crítico elevado teóricamente sufrirán mayores cargas que podrían conducir a un compromiso en la integridad de la reparación, obteniéndose peores resultados funcionales.

La media preoperatoria del ángulo crítico del hombro obtenido $\left(37,1^{\circ}\right)$ es un valor elevado que viene a corroborar lo que ya había sido referido en la bibliografía, desde los estudios iniciales de Moor ${ }^{(6)}$ en los que se describe el mayor riesgo de lesión de manguito rotador en valores altos de este ángulo, hasta los actuales como el de Blon$n a^{(21)}$, en el cual se establece que, mientras que en pacientes control la media era de $34^{\circ}$, en aquellos con roturas del manguito rotador la media era de $36^{\circ}$ en roturas del músculo supraespinoso y de $40^{\circ}$ en roturas de músculos supraespinoso e infraespinoso.

García ${ }^{(19)}$ en su trabajo evaluó la relación entre el ángulo crítico y la rerrotura tras la reparación quirúrgica, sugiriendo la influencia del ángulo crítico en la curación postoperatoria y en la transmisión de cargas, concluyendo que en aquellos pacientes con un ángulo elevado se aumentaba el riesgo de rerrotura completa del manguito y además obtenían peores puntuaciones postoperatorias en la escala americana de cirujanos de hombro y codo. En este aspecto hay que destacar que no siempre las tasas mayores de rerrotura se relacionan con peores resultados en las escalas funcionales.

Por el contrario, Kirsch concluyó que el ángulo crítico no parece ser un predictor significativo en los resultados funcionales y en la puntuación en la escala del dolor tras la reparación quirúrgica de estas lesiones.

En nuestro estudio estadístico existe una asociación significativa entre el tamaño de la rotura y 
el test de Constant preoperatorio y al primer mes. Por lo tanto, las roturas $<3 \mathrm{~cm}$ se relacionan con mejores resultados funcionales preoperatorios y al primer mes.

Sin embargo, no hemos obtenido resultados estadísticamente significativos en la relación entre valores de ángulo crítico menores asociados a mejores resultados funcionales posquirúrgicos, por lo que se refuta la hipótesis inicial.

En cuanto a las limitaciones del estudio, existe un posible factor de confusión en la asociación entre el ángulo crítico y los resultados funcionales, debido a la modificación de la anatomía tras la acromioplastia que podría alterar el curso natural. En los pacientes del estudio se realizó esta técnica de manera protocolizada. Esta modificación en el ángulo fue descrita por Katthagen ${ }^{(22)}$ en cadáveres, de manera que tras la acromioplastia anterolateral se reducía el ángulo crítico una media de 1,40 $y$, si se asociaba con la resección lateral de $5 \mathrm{~mm}$ de acromion, se reducía hasta $2,8^{\circ}$. Sin embargo, concluyen que se necesitan más estudios para discernir si esta reducción es clínicamente significativa. En otro estudio similar de Altintas ${ }^{(23)}$, se obtienen aún mejores resultados mediante la resección lateral artroscópica de $1 \mathrm{~cm}$ del acromion, disminuyendo una media de $6,5^{\circ}$ el ángulo crítico sin discontinuidad macroscópica de la inserción del deltoides, por lo que se necesita estudiar si los pacientes con valores desfavorables del ángulo crítico se beneficiarían de esta técnica.

Además, con un mayor tamaño muestral y un seguimiento más largo, es posible que hubiéramos obtenido un aumento de la significación en los resultados.

La importancia del estudio del ángulo crítico radica en que con la corrección quirúrgica del mismo podríamos prevenir las lesiones degenerativas del manguito o reducir la tasa de rerrotura tras su reparación. Además, podría ayudar a predecir qué pacientes tendrán más riesgo de fracaso tras una artroplastia de hombro por deficiencia del manguito y, por lo tanto, seleccionar a aquellos pacientes candidatos a una artroplastia invertida de hombro.

\section{Conclusiones}

Este estudio coincide con otras publicaciones en las que se confirma la relación existente entre los valores elevados del ángulo crítico en pacientes que han sufrido rotura del manguito rotador.

Existe una asociación significativa entre el tamaño de la rotura y el test de Constant preoperatorio y al primer mes posquirúrgico. Sin embargo, no podemos confirmar que en aquellos pacientes con valores inferiores de ángulo crítico se obtengan mejores resultados funcionales medidos en la escala Constant tras la reparación artroscópica del manguito.

\section{Responsabilidades éticas}

Protección de personas y animales. Los autores declaran que para esta investigación no se han realizado experimentos en seres humanos ni en animales.

Confidencialidad de los datos. Los autores declaran que han seguido los protocolos de su centro de trabajo sobre la publicación de datos de pacientes.

Derecho a la privacidad y consentimiento informado. Los autores declaran que en este artículo no aparecen datos de pacientes.

Financiación. Los autores declaran que este trabajo no ha sido financiado.

Conflicto de interés. Los autores declaran no tener ningún conflicto de intereses.

\section{Bibliografía}

1. Minagawa $H$, Yamamoto $N$, Abe $H$, Fukuda $M$, Seki $N$, Kikuchi K, et al. Prevalence of symptomatic and asymptomatic rotator cuff tears in the general population: From mass-screening in one village. J Orthop. 2013;10(1):8-12.

2. Neer CS. Impingement lesions. Clin Orthop. 1983;173:70-7.

3. Hughes RE, Bryant CR, Hall JM, Wening J, Huston LJ, Kuhn JE, et al. Glenoid inclination is associated with full-thickness rotator cuff tears. Clin Orthop Relat Res. 2003;407:86-91.

4. Wong AS, Gallo L, Kuhn JE, Carpenter JE, Hughes RE. The effect of glenoid inclination on superior humeral head migration. J Shoulder Elbow Surg. 2003;12:360-4.

5. Tétreault P, Krueger A, Zurakowski D, Gerber C. Glenoid version and rotator cuff tears. J Orthop Res. 2004;22:202-7.

6. Moor BK, Bouaicha S, Rothenfluh DA, Sukthankar A, Gerber $C$. Is there an association between the individ- 
ual anatomy of the scapula and the development of rotator cuff tears or osteoarthritis of the glenohumeral joint? A radiological study of the critical shoulder angle. Bone Joint J. 2013;95-B:935-41.

7. Nyffeler RW, Werner CM, Sukthankar A, Schmid MR, Gerber C. Association of a large lateral extension of the acromion with rotator cuff tears. J Bone Joint Surg Am. 2006;88:800-5.

8. Zumstein MA, Jost B, Hempel J, Hodler J, Gerber C. The clinical and structural long-term results of open repair of massive tears of the rotator cuff. J Bone Joint Surg Am. 2008;90:2423-31.

9. Gerber C, Snedeker JG, Baumgartner D, Viehöfer AF. Supraspinatus tendon load during abduction is dependent on the size of the critical shoulder angle: a biomechanical analysis. J Orthop Res. 2014;32:952-7.

10. Viehöfer AF, Gerber C, Favre P, Bachmann E, Snedeker JG. A larger critical shoulder angle requires more rotator cuff activity to preserve joint stability. J Orthop Res. 2016;34:961-8.

11. Moor B, Kuster R, Osterhoff G, Baumgartner D, Werner CM, Zumstein MA, Bouaicha S. Inclination-dependent changes of the critical shoulder angle significantly influence superior glenohumeral joint stability. Clin Biomech (Bristol, Avon). 2016;32:268-73.

12. Moor BK, Wieser K, Slankamenac K, Gerber C, Bouaicha $\mathrm{S}$. Relationship of individual scapular anatomy and degenerative rotator cuff tears. J Shoulder Elbow Surg. 2014;23:536-41.

13. Moor BK, Röthlisberger M, Müller DA, Zumstein MA, Bouaicha S, Ehlinger M, Gerber C. Age, trauma and the critical shoulder angle accurately predict supraspinatus tendon tears. Orthop Traumatol Surg Res. 2014;100:489-94.

14. Pandey V, Vijayan D, Tapashetti S, Agarwal L, Kamath A, Acharya K, et al. Does scapular morphology affect the integrity of the rotator cuff? I Shoulder Elbow Surg. 2016;25:413-21.

15. Suter T, Gerber Popp A, Zhang Y, Zhang C, Tashjian RZ, Henninger $\mathrm{HB}$. The influence of radiographic viewing perspective and demographics on the critical shoulder angle. J Shoulder Elbow Surg. 2015;24:e149-58.

16. Bouaicha S, Ehrmann C, Slankamenac K, Regan WD, Moor BK. Comparison of the critical shoulder angle in radiographs and computed tomography. Skeletal Radiol. 2014;43:1053-6.

17. Spiegl UJ, Horan MP, Smith SW, Ho CP, Millett PJ. The critical shoulder angle is associated with rotator cuff tears and shoulder osteoarthritis and is better assessed with radiographs over MRI. Knee Surg Sports Traumatol Arthrosc. 2016;24:2244-51.

18. Daggett M, Werner B, Collin P, Gauci MO, Chaoui J, Walch G. Correlation between glenoid inclination and critical shoulder angle: a radiographic and computed tomography study. J Shoulder Elbow Surg. 2015;24:1948-53.

19. García GH, Liu JN, Degen RM, Johnson CC, Wong AC, Dines DM, et al. Higher critical shoulder angle increases the risk of retear after rotator cuff repair. J Shoulder Elbow Surg. 2017;26:241-5.

20. Kirsch JM, Nathani A, Robbins CB, Gagnier JJ, Bedi A, Miller BS. Is There an association between the "critical shoulder angle" and clinical outcome after rotator cuff repair? Orthop J Sports Med. 2017; 18;5: 2325967117702126.

21. Blonna D, Giani A, Bellato E, Mattei L, Caló M, Rossi $\mathrm{R}$, Castoldi F. Predominance of the critical shoulder angle in the pathogenesis of degenerative diseases of the shoulder. J Shoulder Elbow Surg. 2016;25:132836.

22. Katthagen JC, Marchetti DC, Tahal DS, Turnbull TL, Millett PJ. The effects of arthroscopic lateral acromioplasty on the critical shoulder angle and the anterolateral deltoid origin: an anatomic cadaveric study. Arthroscopy. 2016;32:569-75.

23. Altintas B, Kääb M, Greiner S. Arthroscopic lateral acromion resection (ALAR) optimizes rotator cuff tear relevant scapula parameters. Arch Orthop Trauma Surg. 2016;136:799-804. 